\title{
Solvent-free ionic liquids as in-situ probes for assessing the effect of ion size on the performance of electrical double layer capacitors
}

\author{
C.O. Ania ${ }^{1}$, J. Pernak ${ }^{2}$, F. Stefaniak ${ }^{2}$, E. Raymundo-Piñero ${ }^{1}$, F. Béguin ${ }^{1 *}$, \\ ${ }^{1}$ CRMD, CNRS-Université, 1 B rue de la Férollerie, 45071 Cedex 2, Orléans, France \\ ${ }^{2}$ Poznań University of Technology, Department of Chemical Technology, pl. Skłodowskiej-Curie 2, \\ 60-965 Poznań, Poland
}

Keywords: porous carbon, electrodes, electrochemical properties

Recently, there is a tremendous interest in increasing energy density of electrochemical capacitors for high-pulse applications, e.g., electric vehicles. In the electrical double layer capacitors (EDLCs), the charges are stored in the electrode/electrolyte interface using highsurface area materials [1]. Activated carbons are the most frequently utilized materials, because they are cheap and they can be produced with a large pore volume, enabling high values of capacitance [1]. However, a good matching between the electrode pore size and the dimensions of the ionic species is critical for an optimal performance of these systems [2-5]. In practice, it is difficult to figure the ideal dimensions of both partners forming the EDL, due to the facts that activated carbons are characterized by a broad pore size distribution and the ions are not all solvated by the same number of solvent molecules.

Moreover, the solvated or non-solvated state of the ions adsorbed in the EDL is not well established. So far, porous carbons have been tentatively used to establish a scale of ionic effective dimensions in aqueous and organic electrolytes, showing that a noticeable proportion of pores might be ineffective for the EDL formation depending on the kind of ions [3-5]. In this work, we propose to circumvent the uncertainty on ion size and their state in the EDL by the use of ionic liquids (ILs). Unlike other studies [7-9], our objective is not to establish the already proven properties of ILs as electrolytes, but to use them for investigating the effect of ion size on the electrosorption mechanism in nanoporous carbons. The great 
advantage of ILs is their ionic nature in the absence of solvent, enabling the effective dimensions of the ions contributing to the formation of the EDL to be reasonably evaluated. Hence, ILs can be used as in-situ probe to explore directly the accessibility of the carbon porous texture during operation of a capacitor. For this purpose, we have prepared phosphonium-derived solvent-free ILs of increasing alkyl chain length.

The phosphonium-based ionic liquids, $\left[\left(\mathrm{C}_{6} \mathrm{H}_{13}\right)_{3} \mathrm{P}\left(\mathrm{ROCH}_{2}\right)\right]\left[\mathrm{Tf}_{2} \mathrm{~N}\right]$, were synthesized following the procedure described elsewhere [10]. In the formula, R stands for the different alkyl chains and $\mathrm{Tf}_{2} \mathrm{~N}$ for the bis(trifluoromethanesulfonyl)imid counteranion. The ILs will be referred to in the text as IL-3, IL-4, IL-7 and IL-12 for R=propyl, butyl, heptyl and dodecyl, respectively. The structure of the ILs is presented in Fig. 1 for clarity. The density and viscosity of ILs were measured with Mettler Toledo DA $110 \mathrm{M}$ scale and micro Ostwald viscometer, respectively. Their conductivity was determined by impedance spectroscopy using a collector|electrolyte|collector stainless steal cell, previously calibrated with standard electrolytic solutions. The physico-chemical characteristics and dimensions of the ILs are given in Table 1.

The electrochemical performance of the composite carbon electrodes in IL electrolytic medium was tested with a two-electrode configuration using stainless-steel swagelok ${ }^{\circledR}$ cells. The activated carbon (AC, from Norit) used for manufacturing the electrodes is characterized by a BET specific surface area of $1653 \mathrm{~m}^{2} / \mathrm{g}$, a large micropore volume (accounting $80 \%$ of the total pore volume) and a well-developed mesoporosity which will favour the accessibility of ions to the micropores [6]. The electrodes are in the form of $1 \mathrm{~cm}^{2}$ pellets obtained by pressing a mixture of carbon material ( $85 \mathrm{wt} . \%)$, acetylene black (5 wt. \%) and polyvinylidene fluoride (PVDF, $10 \mathrm{wt}$ \%). The capacitor device constituted of electrodes of comparable mass (10 to $15 \mathrm{mg}$ ), electrically isolated by a glassy fibrous separator, is assembled in a glove-box under argon. The capacitance properties are studied from 20 to 
$60^{\circ} \mathrm{C}$ by cyclic voltammetry $(1-50 \mathrm{mV} / \mathrm{s})$ and impedance spectroscopy in the frequency range from $100 \mathrm{kHz}$ to $1 \mathrm{mHz}$, using a VMP-2 (Biologic, France). The capacitance values are expressed per mass of activated carbon in one electrode.

Since we want to investigate the effect of alkyl chain length on the capacitor performance, all the measurements have been carried out with pure ILs. As a consequence, the electrochemical characteristics of the capacitors are slightly resistive at room temperature, being this fact linked to the relatively high viscosity and low conductivity of these electrolytes (Table 1). For this reason, electrochemical measurements were also performed at $60^{\circ} \mathrm{C}$, allowing the viscosity to decrease below $100 \mathrm{cP}$.

Fig. 2 shows the impedance spectra of the capacitors at two temperatures for the different electrolytes tested. Increasing temperature from ambient to $60^{\circ} \mathrm{C}$ results in a remarkable decrease of the equivalent series resistance (ESR) for all ILs, e.g., the ESR of IL-3 is 17 and 9 $\Omega$ at 25 and $60{ }^{\circ} \mathrm{C}$, respectively. At high temperature, the viscosity of ILs decreases enhancing the mobility of ions and their penetration in the bulk of the carbon electrode. For comparison, the impedance spectra of $\mathrm{AC}$ based capacitors in aqueous and organic media show that the ESR at $60{ }^{\circ} \mathrm{C}$ in IL-3 is slightly higher than the value obtained with the same carbon in organic medium at room temperature.

Fig. 3 shows the cyclic voltammograms of the capacitors built with the different electrolytes, at room temperature and $60{ }^{\circ} \mathrm{C}$. As expected, the performance of the capacitors increases significantly with temperature due to an enhanced diffusion of ions towards the pores of the carbon electrode. It is noteworthy that an increase of alkyl chain length results in a decrease of capacitance. Since the same trend is observed at both temperatures, it seems reasonable that the size of the cation is crucial for the development of the EDL. However, other factors affecting the EDL capacitance should be taken into account, such as viscosity or conductivity. These two parameters are of the same order for all the electrolytes studied (Table 1). This 
leads to think that the differences in the overall capacitance are unlikely due to differences in the diffusion of the cations inside the pores of the electrode. Moreover, if capacitance would be only related to viscosity, one would expect a variation in the order: IL-7> IL-3 $>$ IL-4 $~$ IL12 according to data from Table 1, whereas the data indicate a link with the ions dimensions. The dimensions of the ions constituting the ILs were evaluated using a computer model (MS Modelling v3.2.0.0) which is a modified version of the Allinger's MM2 force field [11]. The results of the optimized structures showed that the ions do not present a spherical shape. For this reason, only the largest dimension of the ionic species was estimated. As expected, the cation size increases with the length of the alkyl chain, from 0.8 to $2 \mathrm{~nm}$ (Table 1), being much larger than the estimated for the counteranion, bis(trifluoromethylsulfonyl)imid (0.64 $\mathrm{nm})$. Taking into account the series dependence of the overall capacitance of a two-electrode cell [1], we assume that this value is controlled by the electrode where the cations are sorbed, since the counteranion has a much smaller size than the former, and is kept constant for all the ionic liquids.

Accordingly to the size of the cations herein calculated, the pores smaller than $0.8 \mathrm{~nm}$ are not accessible to any of the electrolytes tested. In agreement with the pore size distribution of the activated carbon obtained from the $\mathrm{N}_{2}$ adsorption data, this represents $39 \%$ of the overall porosity which cannot contribute to the formation of the EDL, and thus to the capacitance of the negative electrode. It can then be concluded that the porosity of this electrode is underused from an electrochemical point of view. Micropores between $0.8-2 \mathrm{~nm}$ still represent $42 \%$ of the total porosity, although they would be all accessible only in the case of IL-3. As long as the alkyl chain increases (the largest dimension of the cation), the volume of pores accessible to the electrolyte is smaller and the capacitance value decreases. The volume fraction of AC corresponding to micropores larger than the cation size was estimated by applying the DFT model to the nitrogen adsorption data, and represented versus the capacitance values (Fig. 4). 
The figure shows almost a proportional dependence of capacitance with the so-defined accessible volume, confirming the outstanding role of the relationship between pore size and cation size on the formation of the EDL. Considering that activated carbons are constituted of slit-shape pores, among the two extreme orientations of the cations chains versus the pore walls, i.e. parallel or perpendicular, our results indicate that the perpendicular position is preferred. A similar situation has already being reported for the intercalation of quaternary ammonium derivatives in lamellar hosts [12], where the ions are perpendicular to the layers presenting their positive charges close to the negative ones located on these layers.

In summary, the sieving effect of the carbon host in the formation of the electrical double layer was clearly demonstrated by using solvent-free ILs of increasing cation dimensions. Taking into account the textural properties of carbon evaluated by gas adsorption and the size of the electrolytes calculated by molecular modelling, it was proved that the overall porosity of the carbon electrode is underused.

Contrarily to previous papers reporting that pores within the range of 0.8-2 nm together with a moderate amount of mesopores $[1,2,5]$ mainly contribute to the EDL formation, our results indicate that there is not an optimum pore size of the electrode material. Hence, the performance of capacitors can be only optimized if the porosity of the carbon electrode and the dimensions of the chosen electrolyte match to each other. The dependence of capacity values with cations dimensions confirms that the perpendicular position of the chains is preferred. This information is of extremely relevant importance for a fundamental understanding of the EDL formation in porous carbons.

\section{Acknowledgements}

The authors thank Norit for kindly supplying the activated carbon adsorbent. COA thanks MEC, Spain, for the financial support (EX2004-0612). Poznań University of Technology is 
also acknowledged (Project DS 32/007/2005). The help of Dr. Parra with the Modelling Software and of Dr. Khomenko in the conductivity measurements is acknowledged.

\section{References}

[1] Frackowiak E, Béguin F. Carbon materials for the electrochemical storage of energy in capacitors. Carbon 2001; 39(6): 937-950

[2] Kim YJ, Horie Y, Ozaki S, Matsuzawa Y, Suezaki H, Kim C, et al. Correlation between the pore and solvated ion size on capacitance uptake of PVDC-based carbons. Carbon 2004; 42(8-9):1491-1500

[3] Salitra G, Soffer A, Eliad L, Coher Y, Aurvach D. Carbon electrodes for double-layer capacitors- I. Relations between ion and pore dimensions. J Electrochem Soc 2000; $147: 2486-2493$

[4] Eliad L, Salitra G, Soffer A, Aurbach D. Ion sieving effects in the electrical double layer of porous carbon electrodes: Estimating effective ion size in electrolytic solutions J Phys Chem B 2005; 105:6880-6887

[5] Lin Ch, Ritter JA, Popov BN. Correlation of Double-Layer Capacitance with the Pore Structure of Sol-Gel Derived Carbon Xerogels. J Electrochem Soc 1999; 146:3639-3643

[6] Yoon S, Lee J, Hyeon T, Oh SM. Electric Double-Layer Capacitor Performance of a New Mesoporous Carbon. J Electrochem Soc 2000; 147:2507-2512

[7] Frackowiak E, Lota G, Pernak J. Room-temperature phosphonium ionic liquids for supercapacitor application. Appl Phys Letters 2005; 86:165104

[8] Suarez PAZ, Selbach VM, Dullius JEL, Einloft S, Piatnicki CMS, Azambuja DS, et al. Enlarged electrochemical window in dialkyl-imidazolium cation based roomtemperature air and water-stable molten salts. Electrochim. Acta 1997; 42: 2533-2535

[9] Balducci A, Bardi U, Caporali S, Mastragostino M, Soavi F. Ionic liquids for hybrid supercapacitors. Electrochem Comm 2004; 6:566-570 
[10] Pernak J, Stefaniak F, Węglewski J. Phosphonium acesulfamate based ionic liquids. Eur. J Org Chem 2005; 4:650-652

[11] Kao J, Allinger NL. Conformational-analysis.122. Heats of formation of conjugated hydrocarbons by force-field method. J Am Chem Soc 1977; 99:975-986

[12] Béguin F, Rouzaud JN, Maimoun IB, Seron A. Elaboration and structure of silicate/carbon nanocomposites. J Phys Chem Solids 1996; 57:1019-1029 
Table 1. Dimensions and physico-chemical characteristics of the series of phosphoniumderived ILs at $25^{\circ} \mathrm{C}$.

\begin{tabular}{ccccc}
\hline Nomenclature & $\begin{array}{c}\text { Density } \\
{\left[\mathrm{g} / \mathrm{cm}^{3}\right]}\end{array}$ & $\begin{array}{c}\text { Viscosity } \\
{[\mathrm{cP}]}\end{array}$ & $\begin{array}{c}\text { Conductivity } \\
{[\mathrm{mS} / \mathrm{cm}]}\end{array}$ & $\left.\begin{array}{c}\text { Largest } \\
\text { dimension } \\
(\AA)\end{array}\right)^{*}$ \\
\hline IL-3 & 1.122 & 106.4 & 0.51 & 8.5 \\
IL-4 & 1.112 & 132.9 & 0.33 & 8.9 \\
IL-7 & 1.074 & 97.6 & 0.32 & 11.5 \\
\hline
\end{tabular}

* Computed from the MS modelling software 
Figure 1. Molecular structure of the phosphonium-based cations obtained from the MSmodelling software.
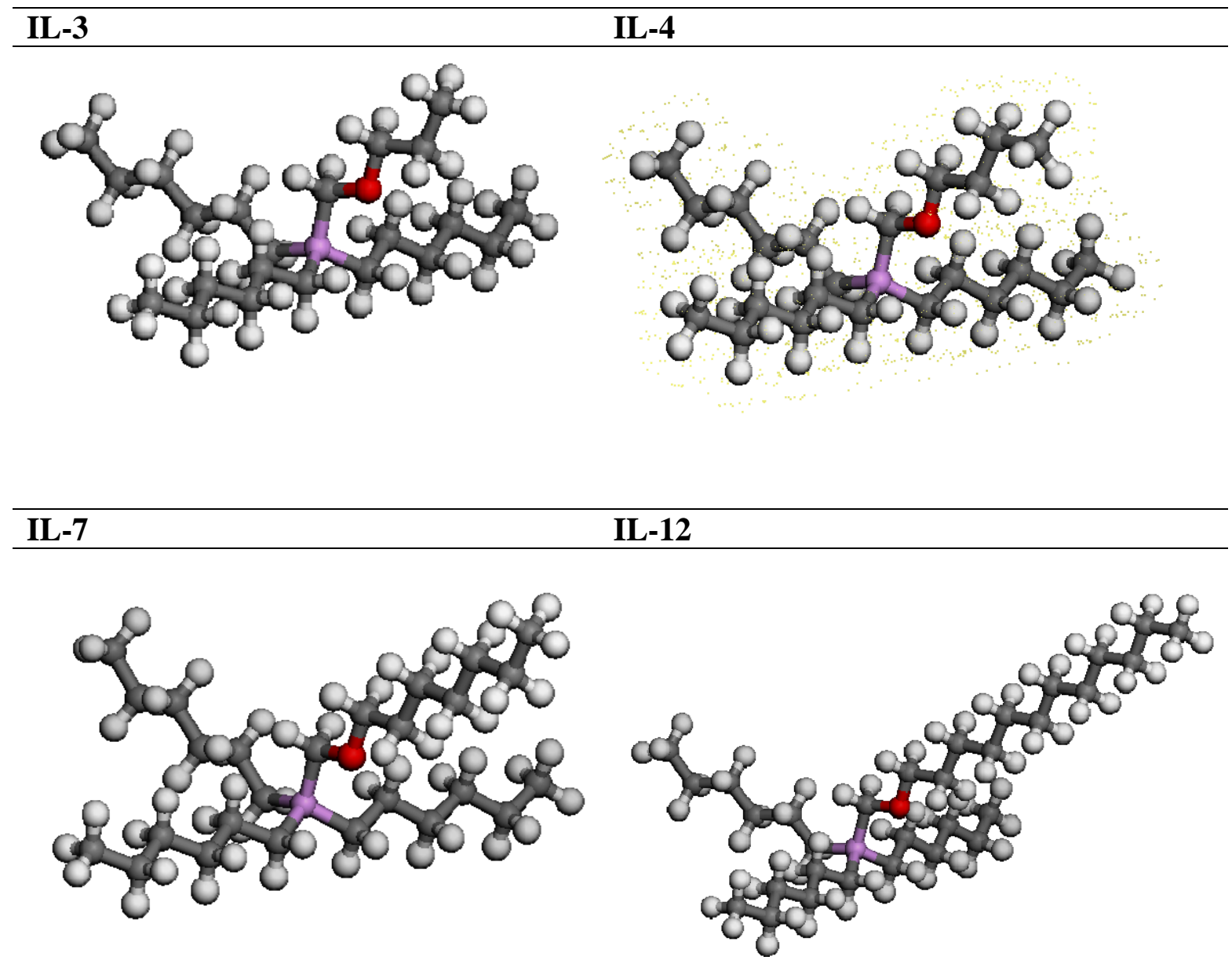
Figure 2. Nyquist plots of supercapacitors built from AC and phosphonium-based ILs at: A) room temperature; B) $60{ }^{\circ} \mathrm{C}$. Inset: Impedance spectra of the carbon electrodes in acidic $\left(\mathrm{H}_{2} \mathrm{SO}_{4}, 1 \mathrm{~mol} . \mathrm{L}^{-1}\right)$ and organic media $\left(\mathrm{TEABF}_{4}, 1 \mathrm{~mol} . \mathrm{L}^{-1}\right.$ in acetonitrile). Frequency range from $100 \mathrm{kHz}$ to $1 \mathrm{mz}$.
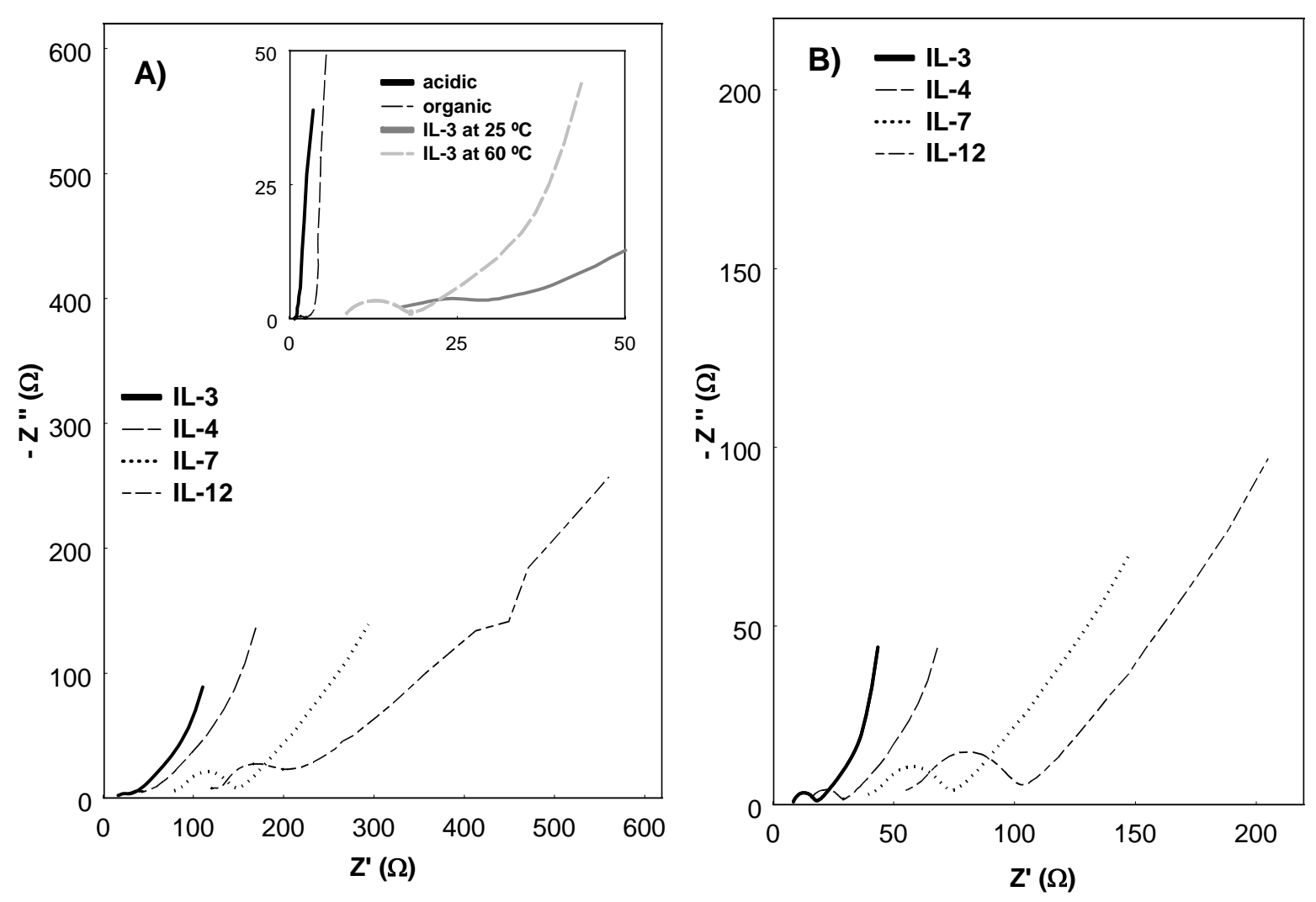
Figure 3. Voltammetry characteristics $(2 \mathrm{mV} / \mathrm{s})$ of the activated carbon $\mathrm{AC}$ in the solvent-free solutions of the different ionic liquids, at: A) room temperature; B) $60^{\circ} \mathrm{C}$.
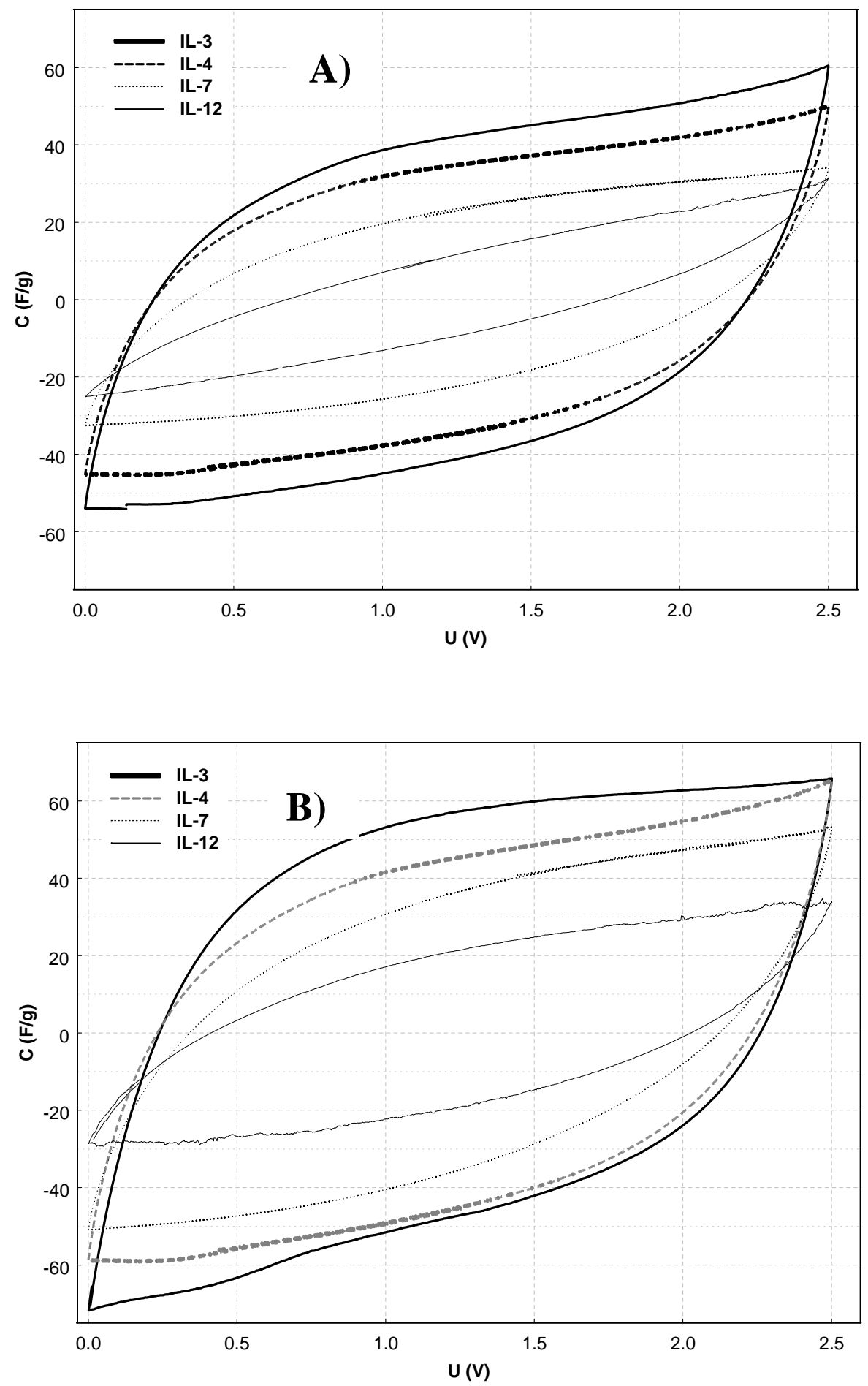
Figure 4. Relationship between the cation size or the accessible micropore volume of the AC electrode and the capacitance values.

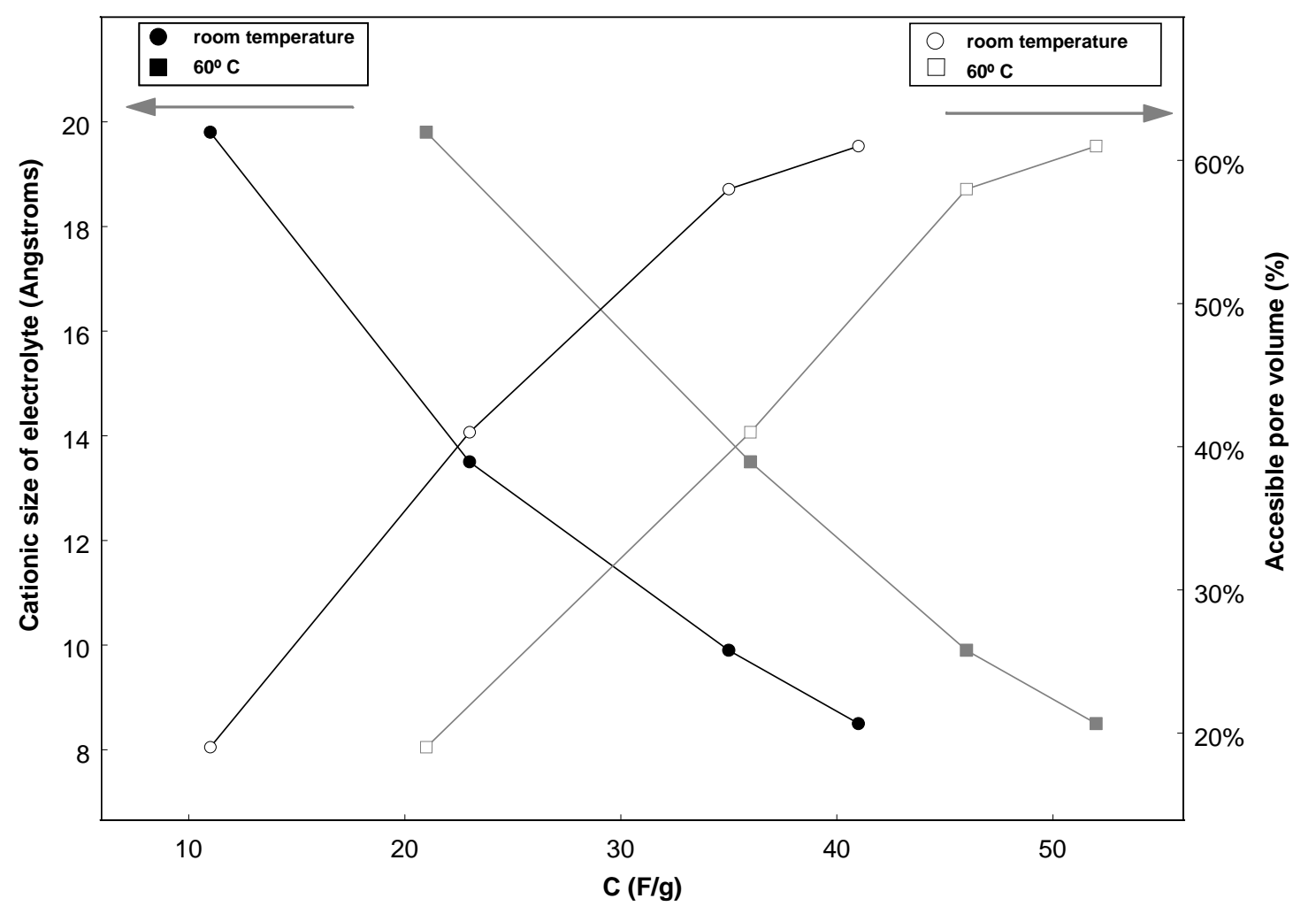

